\title{
Primary Malignant Epithelioid Hemangioendothelioma of the Liver in a Young Man
}

\author{
Mamatha Siricilla ${ }^{1}$, Khalid Rasheed ${ }^{1 *}$ and Robert A Avery ${ }^{2}$
}

${ }^{1}$ Department of Medicine, University of Alabama at Birmingham, Montgomery Internal Medicine Residency Program, USA

${ }^{2}$ Division of Hematology and Oncology, Cancer Care Center of Montgomery - Jackson, USA

\begin{abstract}
Due to lack of experience of clinicians, radiologists and histopathologists, owing to the paucity of cases of Hepatic Epithelioid Hemangio endothelioma [HEHE], identification of this entity is not easy. For these reasons these patients are being diagnosed late or misdiagnosed. Young adults with no prior history of chronic liver disease with subtle laboratory parameters, characteristic imaging findings, absence of tumor markers is indicative of HEHE, as early diagnosis and treatment significantly improves survival rate.
\end{abstract}

Keywords: Hepatic endothelioma; Calcified hepatic mass; Metastasis

\section{Case Report}

A 38-year-old Asian man presented with four months history of nonproductive cough, shortness of breath, and back pain. Routine blood tests revealed abnormal liver function (aspartate aminotransferase level, $58 \mathrm{U} / \mathrm{L}$ [normal 10-40]; alanine aminotransferase level, 136 U/L [normal 0-40]; alkaline phosphatase level, 211 U/L [normal 53128] and gamma-glutamyl transferase level, $167 \mathrm{U} / \mathrm{L}$ [normal 0-50]. Serologic tests for hepatitis serology for A, B and C were negative. Computerized Tomography (CT) of the abdomen and chest revealed a $4.5-\mathrm{cm}$ mass in the right lobe of the liver (Figure $1 \mathrm{~A}$ and $\mathrm{B}$ ) and bilateral reticular lung nodules suggestive of metastasis (Figure 2C). Bronchial washings were negative for tuberculosis and malignancy. Magnetic resonance imaging of the thoracic and lumbar spine revealed an abnormal signal from T8 to L2, indicating metastasis (Figure $2 \mathrm{D}$ ). Tumor markers alpha-fetoprotein, Carbohydrate antigen 19-9 (CA 199) and Carcinoembryonic Antigen (CEA) were all within the normal range.

Liver biopsy demonstrated areas of perivascular atypical epithelioid and spindle cells (Figure 3E maroon arrows) within the myxoid and fibrotic stroma (Figure 3E, orange arrows) with mild to moderate nuclear atypia (Figure 3F, black arrows). Immunohistochemistry showed positivity for the vascular markers CD31 (cytoplasmic) and ERG (nuclear), supporting the diagnosis of Hepatic Epithelioid Hemangioendothelioma (HEH). As the patient had metastatic disease with involvement of liver, bone and lungs, chemotherapy with cyclophosphamide and prednisone two week cycle was initiated. After two months of treatment patient developed respiratory failure secondary to recurrent pleural effusion complicated by pneumonia and acute respiratory distress syndrome. There was no improvement of the metastatic lesions as shown by the CT scan. Trial of Paclitaxel was begun, but the patient's condition further worsened within the following few weeks and died 4 months from the initial diagnosis.

\section{Discussion}

Primary malignant epithelioid hemangioendothelioma of the liver is a rare tumor with an incidence of fewer than 0.1 cases per 100,000 individuals per year and arises in patients without a prior history of chronic liver disease [1]. The disease occurs most often in young, healthy people in the second and third decades of life and is more common in females than in males (the ratio of cases in females to cases in males is 3:2) [2]. The differential diagnosis includes angiosarcoma, cholangiocarcinoma, fibrolamellar hepatocellular carcinoma and metastatic carcinoma [2]. In general, the clinical course is intermediate between hemangioma and hemangioendotheliosarcoma. Previous reports of clinical outcome range from spontaneous regression to death within months after diagnosis.

On ultrasonography, a hypoechoic mass is usually seen. Computerized tomography findings suggestive of hepatic epithelioid hemangioendothelioma include a large hypodense mass located mainly in the periphery of the liver with calcifications and peripheral enhancement and a tendency of the tumor nodules to merge into each other [2]. Magnetic resonance imaging reveals a hypointense mass on T1-weighted images and a hyperintense mass on T2-weighted images [1].

Definitive diagnosis requires histopathological and immunohistochemical evidence of endothelial differentiation with positive staining for endothelial cell markers like CD31 (platelet endothelial cell adhesion molecule 1), CD34 (human hematopoietic progenitor cell antigen), and factor VIII-related antigen [1] Podoplanin, which is immunoreactive to D2-40 antibody and ERG are new promising and highly specific markers for vascular endothelial tumors $[3,4]$.

Treatment varies with the extent of disease, presentation and local expertise with various therapeutic modalities. In patients with only liver involvement, surgical resection has promising results, as has orthotopic liver transplantation. Unfortunately, most cases are multicentric or metastatic at presentation, and such tumors often tend to be aggressive and have poor prognosis [5]. For metastatic disease to date there are no validated therapeutic options. In literature there have been case reports with variable success using different treatment modalities including chemotherapeutic agents, endovascular embolization, and radiation therapy. Recently metronomic cyclophosphamide has been proposed as a new palliative therapeutic option to treat metastatic

*Corresponding author: Khalid Rasheed, Department of Internal Medicine University of Alabama At Birmingham Health Center Montgomery, Alabama 36116 USA, E-mail: khalid_rashid24@yahoo.co.in

Received July 05, 2013; Accepted September 04, 2013; Published September 13,2013

Citation: Siricilla M, Rasheed K, Avery RA (2013) Primary Malignan Epithelioid Hemangioendothelioma of the Liver in a Young Man. J Liver 2: 126 doi:10.4172/2167-0889.1000126

Copyright: @ 2013 Siricilla M, et al. This is an open-access article distributed under the terms of the Creative Commons Attribution License, which permits unrestricted use, distribution, and reproduction in any medium, provided the original author and source are credited. 


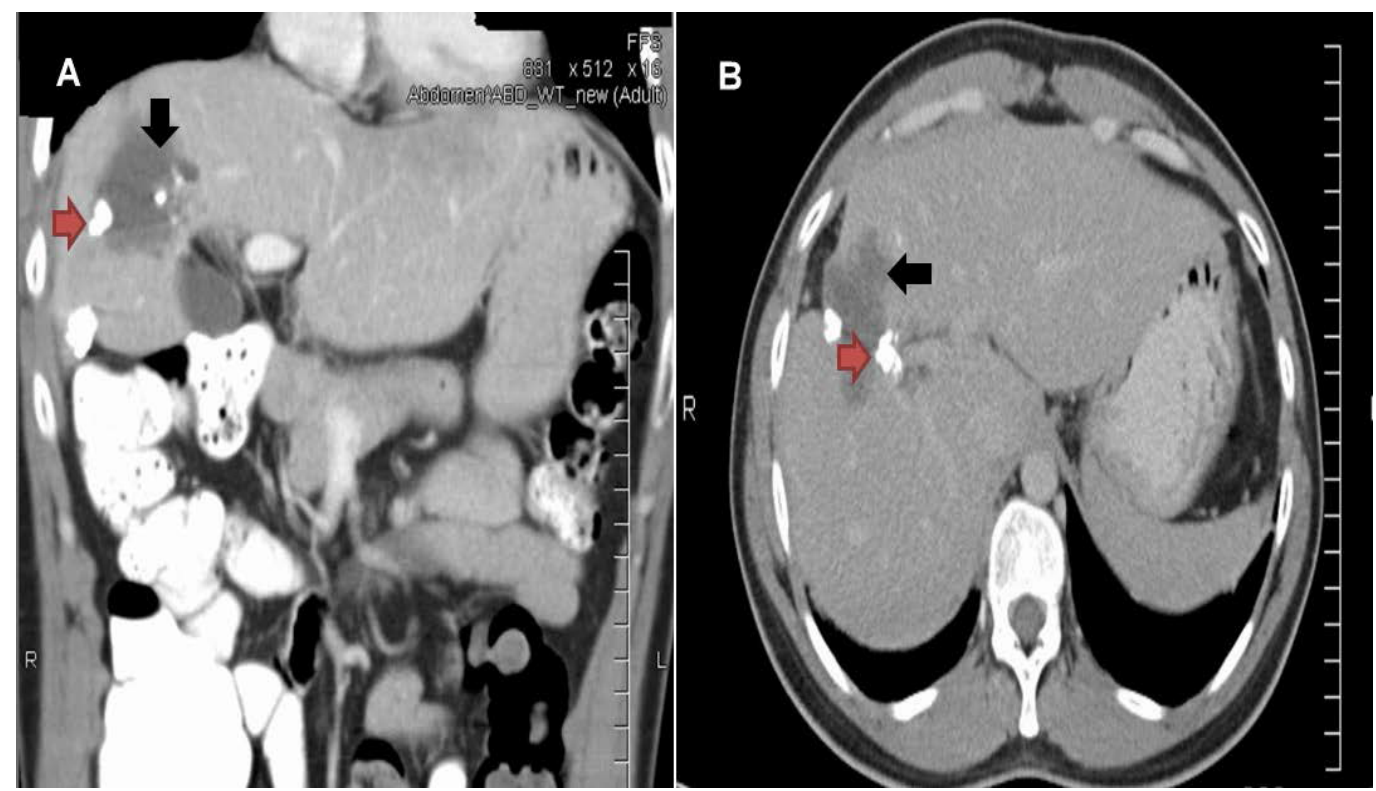

Figure 1(A \& B): Computerized Tomography (CT) of the abdomen and chest revealed a $4.5-\mathrm{cm}$ mass in the right lobe of the liver.

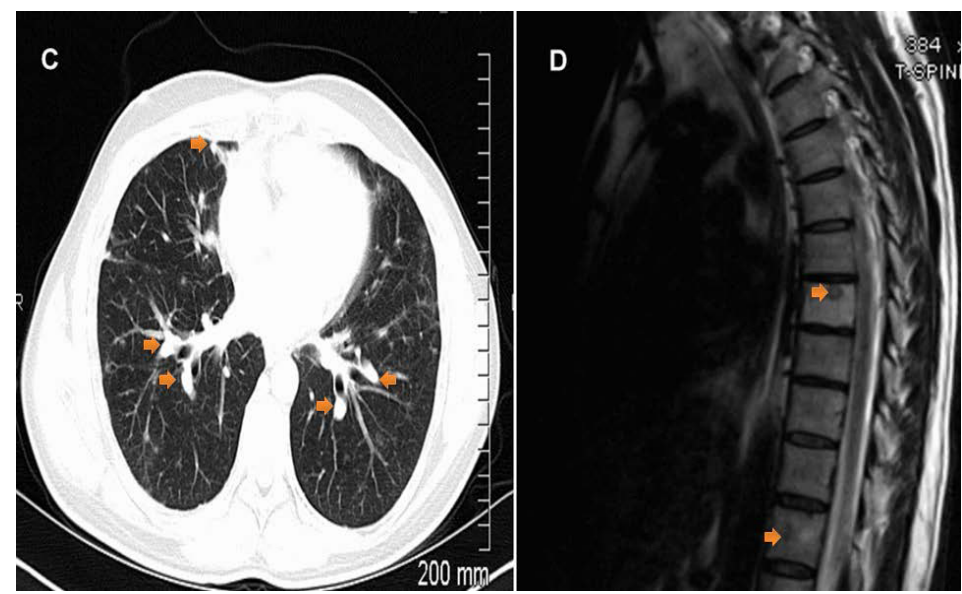

Figure 2: (C)Bilateral reticular lung nodules suggestive of metastasis. (D) Magnetic resonance imaging of the thoracic and lumbar spine revealed an abnormal signal from $\mathrm{T} 8$ to $\mathrm{L} 2$, indicating metastasis.

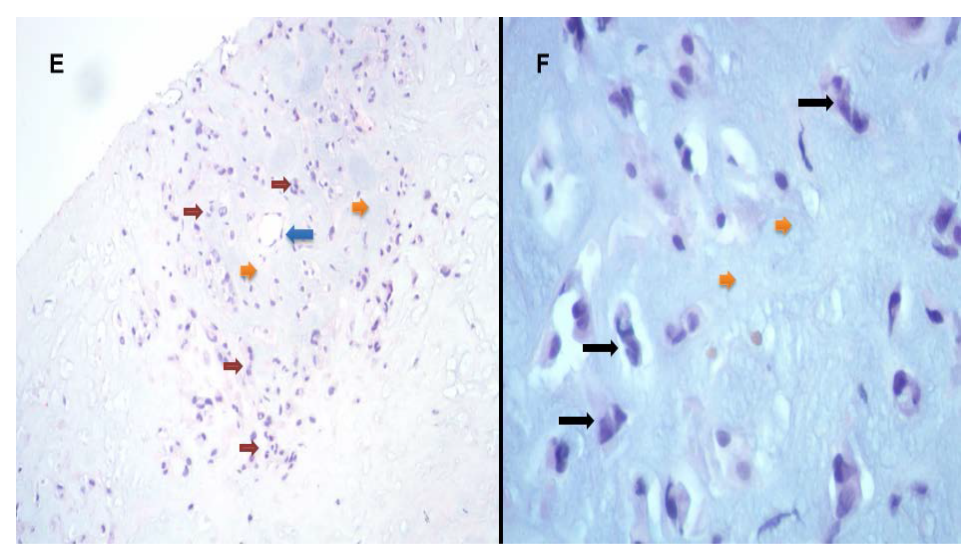

Figure 3: (E) Liver biopsy demonstrated areas of perivascular atypical epithelioid and spindle cells (maroon arrows) within the myxoid and fibrotic stroma (orange arrows). (F) Liver biopsy demonstrated areas of perivascular atypical epithelioid and spindle cells with mild to moderate nuclear atypia (black arrows). 
Citation: Siricilla M, Rasheed K, Avery RA (2013) Primary Malignant Epithelioid Hemangioendothelioma of the Liver in a Young Man. J Liver 2: 126. doi:10.4172/2167-0889.1000126

Page 3 of 3

HEH [6]. Bevacizumab, thalidomide and interferon have also been used for treatment HEH again with variable success. Mehrabi et al. have proposed liver transplant and chemotherapy or trans arterial chemo embolization and chemo radiation for diffuse liver disease with extrahepatic involvement [1]. Prognosis of hepatic HEH is highly variable and sometimes independent of the extent of the disease at presentation [5]. However, it is difficult to assess the effectiveness of each therapy and to decide on the therapeutic strategy for HEH because of the rarity and unpredictable behavior of this neoplasm. Presented case has diffuse metastatic disease at presentation and cyclophosphamide was not successful in treating the disease. Reporting such cases with low incidence will help us understand the natural course and tumor behavior to various treatment strategies.

\section{References}

1. Mehrabi A, Kashfi A, Fonouni H, Schemmer P, Schmied BM, et al. (2006) Primary malignant hepatic epithelioid hemangioendothelioma: a comprehensive review of the literature with emphasis on the surgical therapy. Cancer 107: 2108-2121.

2. 2. Akça S, Süleymanlar I, Dinçer D, Demirbaş A, Gelen T, et al. (2002) Hepatic epithelioid hemangioendothelioma treated with orthotopic liver transplantation: a case report. Turk J Gastroenterol 13: 221-225.

3. Miettinen M, Wang ZF, Paetau A, Tan SH, Dobi A, et al. (2011) ERG transcription factor as an immunohistochemical marker for vascular endothelial tumors and prostatic carcinoma.Am J Surg Pathol 35: 432-441.

4. Fujii T, Zen Y, Sato Y, Sasaki M, Enomae M, et al. (2008) Podoplanin is a useful diagnostic marker for epithelioid hemangioendothelioma of the liver. Mod Pathol 21: 125-130.

5. Oshima N,TerajimaH, Hosotani R (2009) Surgical Therapy for a Solitary form of Hepatic Epithelioid Hemangioendothelioma:A Long-Term Survival Case. Case Rep Gastroenterol 3: 214-221

6. Lakkis Z, Kim S, Delabrousse E, Jary M, Nguyen T, et al. (2013) Metronomic cyclophosphamide: an alternative treatment for hepatic epithelioid hemangioendothelioma. J Hepatol 58: 1254-1257. 